\title{
Reception frequency bandwidth of a gravitational resonant detector with optical readout
}

\author{
A.V.Gusev, V.N.Rudenko, S.A.Cheprasov $\dagger$ \\ M.Bassan $\ddagger$ \\ $\nmid$ †AI. MSU, Moscow, Russia; \\ $\ddagger$ Dip. Fisica, Università Tor Vergata and INFN Roma, Italy
}

\begin{abstract}
A gravitational resonant bar detector with a large scale Fabry-Perot cavity as an optical read out and a mechanical displacement transformer is considered. We calculate, in a fully analytical way, the final receiver bandwidth in which the potential sensitivity, limited only by the bar thermal noise, is maintained despite the additional thermal noise of the transformer and the additive noise of the optical readout. We discuss also an application to the OGRAN project, where the bar is instrumented with a $2 \mathrm{~m}$ long FP cavity.
\end{abstract}

Submitted to: Class. Quantum Grav.

PACS numbers: 0480N,0760 


\section{Introduction}

A search for a new type of cosmic radiation - gravitational waves, - has been carried out during last years with two kinds of gravitational wave detectors: cryogenic resonant bars [1] and laser interferometers on suspended mirrors [2]. Bars have already been in operation for more than ten years. Meanwhile interferometers are beginning their active "scientific life". Starting with year 2008 a series of incremental upgrades of the interferometers is planned. In the next five years there will be long periods when most of these observatories will be out of operation to complete such upgrades, while resonant bars will remain in scientific duty, accumulating observational data. The great reliability and the low cost of operation, demonstrated in many years of observations, make it reasonable and worthwhile to keep resonant detectors on the air as "supernova watch" during these coming years. Their sensitivity has proven to be accurately predictable, on the basis of detector dynamics and fundamental noise sources, with the possible exception of some excess in the high energy tail of the event distribution.

A clear advantage of the "free mass interferometer GW detector" consists in its wide detection frequency band $\sim 10^{3} \mathrm{~Hz}$. However this band is not a homogeneous low noise region, being composed of a number of "windows of good sensitivity" separated by peaks of increased noise associated with resonances of suspensions and other technical causes. The typical width of such window is on order of $\sim 100 \div 300 \mathrm{~Hz}$. The best sensitivity achieved at present inside the window in the region a few hundred $\mathrm{Hz}$ is characterized by the noise spectral density $\sim 3 \cdot 10^{-23} \mathrm{~Hz}^{-1 / 2}$; in the zone of the bar vision $(1 \pm 0.2) k H z$ noises are somewhat higher $\sim 1 \cdot 10^{-22} \mathrm{~Hz}^{-1 / 2}$ ) and growing linearly with frequency in terms of metric perturbations [2].

The present generation of "cryogenic resonant bar detectors" was designed for cooling below $100 \mathrm{mK}$ : however they actually operate at the temperature $\sim(2 \div 3) \mathrm{K}$ in the " $\mathrm{kHz}$ " region reaching almost the same noise spectral density $\sim 5 \cdot 10^{-22}, \mathrm{~Hz}^{-1 / 2}$ but in a very narrow frequency band $\sim(1 \div 0.1) \mathrm{Hz}$. In recent years, all cryogenic bar groups have modified their read-out systems in order to achieve a larger bandwidth $(20 \div 100$ $\mathrm{Hz}$ ). This was achieved with a small loss in peak sensitivity, now in the $10^{-21} \mathrm{~Hz}^{-1 / 2}$ range (that is however by no mean fundamental: it is mainly due to the lower $\mathrm{Q}$ factor of high coupling devices and could be regained in possible future upgrades).

The limitation of the bar reception bandwidth arises from noises of the read out, but not from the thermal fluctuation of the bar itself. Indeed, the potential sensitivity of the bar cooled down to the temperature $10 \mathrm{mK}$ and equipped by a noiseless read out sensor corresponds to its thermal noise spectral density $\sim 10^{-23} \mathrm{~Hz}^{-1 / 2}$, i.e to the noise level projected for advanced interferometers at $1 \mathrm{kHz}$. A clear understanding of this fact stimulates the "bar groups" for an investigation of new types of low noise recording devices. In that number the fist place belongs to the "optical read out" which in fact was successfully used in the interferometers to measure the displacements of test mass-mirrors [2].

In this paper we analyze reception characteristics (the sensitivity and bandwidth) 
of a gravitational bar detector with mechanical transducer (displacement transformer) and an optical FP-cavity as a read out system. As an intermediate step we consider a possibility of using a displacement transformer as a matching link between the bar and FP-cavity at room temperature (the Russian project "OGRAN" [3]). However our final target is a calculation of "the maximum receiver bandwidth" for the "super cryogenic bar" $(10 \mathrm{mK})$ with optical read out at the level of its potential sensitivity. We have carried out our analysis considering as background noise only that of unavoidable natural fluctuation: thermal noises of mechanical parts and shot noise in optical channels. Non fundamental limitation of technical noises is discussed briefly in a comparison with experiments performed in recent years.

\section{Equivalent scheme and notations}

All existing gravitational bar detectors utilize the displacement transformer (DT) - a light mechanical oscillator attached to one end of the aluminum bar, for an impedance matching of the bar acoustical resonator to the EM-sensor (which is usually part of a LC resonance circuit + DC SQUID for cryogenic detectors) [4], [5], 6]. Mechanical construction of DT was realized in the form of "membrane", "loaded diaphragm", "mushroom" or "rosette". At the resonance condition $\omega_{\mathrm{Bar}}=\omega_{\mathrm{DT}}=\omega_{0}$ the energy of bar's oscillation is transferred to DT with the "beating frequency" $\Omega_{B}=0.5 \sqrt{\mu} \omega_{0}$, where the small parameter $\mu=m / M \ll 1$ is defined by the ratio of DT and bar masses. Under a good matching, the DT amplitude is larger by the factor $\mu^{-1 / 2}$ than the bar amplitude.

A general theory of the bar with DT was initially published in [7], [8] and then elaborated in many papers of "bar groups". A principal role of the optical read out for the classical Weber bar was theoretically investigated in the paper [9]. Afterwards, this idea was developed in [10] and then a pilot model was designed and tested at room temperature [11, [13], [12]. The possibility to achieve the sensitivity $10^{-20} \mathrm{~Hz}^{-1 / 2}$ for a room temperature bar with a good optical readout have been shown in [14]. The correctness of this estimate was demonstrated in the paper [15] where the first full implementation of a bar with opto-mechanical readout was constructed and tested.

There are at least two ways to couple a bar with an optical FP-cavity. In both cases one mirror of the cavity must be clamped to the membrane of DT. For the second mirror one has a choice: it may be attached either to the front (near) or to the opposite (far) bar's end surface. For conventional bars [4], [5], [6] mainly the front end can be used. In this variant DT together with FP-cavity presents a single measuring element - a sensitive accelerometer. To improve the coupling and to suppress optical noises the FP-cavity gap must be as small as possible [12], [15]. A direct action of GW on such accelerometer produces much smaller perturbation in comparison with the bar's reaction and so it may be neglected.

A different situation arises if the second mirror can be attached to the far end of

the bar. Such opportunity is indeed available in the Russian project OGRAN, in which 
the cylindrical bar detector has an optical tunnel along its central axis [3]. In this configuration the "electromagnetic degree of freedom" (long optical FP resonator) has the same geometrical scale as the acoustical one. It means that a "GW-reaction" of the electromagnetic part of the detector has to be taken into account on equal footing with the acoustical one. Actually, as it was shown in the paper [9], a reaction of such "opto-acoustical" gravitational detector contains, in general, two separate parts: the conventional acoustical response and a new one, the optical response. However, in a "long wave limit" (when the GW wavelength is much larger the bar length) the optical response of a high finesse FP-cavity is small enough and, in the first approximation, may be neglected.

In our analysis below we will consider namely the second nontraditional configuration: a bar with a long FP-cavity; one mirror is attached to the far bar end face, and the other to a DT fastened to the opposite bar end. Using a reference system with the origin in the bar center one can present an equivalent scheme of the opto-acoustical detector as it is shown in fig.1.

Additional notations used in this figure are the following. $k_{1}=M \omega_{1}^{2}, k_{2}=m \omega_{2}^{2}$ - coefficients of rigidity, masses and partial frequencies of the bar and DT with corresponding coordinates $x_{1}, x_{2}$. Coefficients of losses are $H_{1}, H_{2}$ (we assume a frictional force, proportional to velocity of the mass). The eigen frequencies of the "coupled oscillatory system" are defined as $\omega_{e 1}=\omega_{0}-\Omega_{B}, \Omega_{e 2}=\omega_{0}+\Omega_{B}$.

One can see the first mirror of FP cavity is fixed at the point $x=0$, the second moving mirror is clamped to the DT mass. The auxiliary optical components - laser, beam splitter and photo receiver, are symbolically shown in fig.1. In the hypothesis of constant laser power $P$ and frequency $\omega_{e}$ such configuration presents the typical scheme of a parametric read out: the signal of interest being detected through variations of some parameter, namely — the length of FP cavity.

Arrows represent all forces of our interest: the equivalent GW action is represented by a "signal force" $F_{s}$; the thermal excitation are symbolically shown through "Langevin stochastic forces" $F_{f l 1}, F_{f l 2}$.

Having in mind the model of fig. 1 we can easily write down the equations of motion, which in general describe a dynamics of three coupled degree of freedom: bar, DT and FP cavity. Below we will consider a special case of our interest with a resonance optical pump. In this case, the bar acoustical oscillation produces only a phase modulation of the output light; its residual amplitude variation may be neglected [16]. This means that the dynamical influence of the FP cavity on the bar is absent: there is no "optical spring" effect [17]. Besides, one can omit also the "back action fluctuation" effect if the pump power is smaller the critical one, which is extremely large $P_{c r} \ll 1 \mathrm{~kW}$ (see Appendix).

In this approximation the third equation, describing the FP cavity, is reduced to a simple factor of conversion of a "DT mechanical displacement" into the "optical output signal". However a specific electromagnetic or "read out noise" has to be added to acoustical thermal noises. 
After this remarks we will write down the equations of motion and will analyze a solution to estimate the detection sensitivity and receiver bandwidth.

\section{Equations of motion and noise spectrum}

A signal track block diagram is shown in fig.2. GW-signal and thermal fluctuation are represented by their equivalent forces reduced to the input of the acoustical part of the GW detector (bar + DT). The output of this part,- a DT coordinate perturbation $x_{D T}=x_{2}(t)$, produces variations of the FP cavity length $L(t)=L_{0}+x_{2}(t)+x_{1}(t)$; being $x_{2} \gg x_{1}$, we neglect the last term. This length change leads to phase modulation of the output light, recorded by a heterodyne photo detector (HPD). The proper noise of such photo detection (just a "read out noise") has to be modeled in a general SNR balance.

In this paper we would like to avoid further detailing of the block diagram fig.2 keeping in the mind different conceivable schemes of optical demodulation such as the self photo detection type of the Pound-Drever technique [15] or the use of some external reference optical flux at the heterodyne photo mixer [19].

Introducing the differential operator $p=d / d t$ one can write the equations of motion of the acoustical part in algebraic form. The losses are taken into account through the well known formalism of "complex rigidity": $\tilde{k}_{i}=k_{i}+p H_{i}[18$.

$$
\left\{\begin{array}{c}
{\left[M p^{2}+p\left(H_{1}+H_{2}\right)+\left(k_{1}+k_{2}\right)\right] x_{1}-\left(k_{2}+p H_{2}\right) x_{2}=F_{s}+F_{f l 1},} \\
\left(m p^{2}+p H_{2}+k_{2}\right) x_{2}-\left(k_{2}+p H_{2}\right) x_{1}=F_{f l 2}
\end{array}\right.
$$

A solution of these equations for the DT coordinate is written as

$$
\left\langle x_{2}(t)\right\rangle=K_{m}(p) F_{s}(t)
$$

where

$$
\begin{gathered}
K_{m}(p)=\frac{k_{2}+p H_{2}}{\operatorname{det}(p)} \\
\operatorname{det}(p)=\left[M p^{2}+p\left(H_{1}+H_{2}\right)+\left(k_{1}+k_{2}\right)\right]\left(m p^{2}+p H_{2}+k_{2}\right)-\left(k_{2}+p H_{2}\right)^{2} .
\end{gathered}
$$

For $H_{1}=H_{2}=0$ one has

$$
\begin{aligned}
& \operatorname{det}(p) \propto\left(p^{2}+\omega_{e 1}^{2}\right)\left(p^{2}+\omega_{e 2}\right), \\
& \left(\omega_{e 1}+\omega_{e 2}\right) / 2=\omega_{0} \approx \omega_{e 1}, \omega_{e 2} .
\end{aligned}
$$

Having the signal variable $x_{2}(t)$, as a next step one can calculate the reaction of the resonating FP cavity to variation of its length $L=L_{0}+x_{2}$. It will be in the form of a phase modulated transmitted light. Such signal in particular might be extracted through a heterodyne photodetection procedure accompanied by the "Mandel photo current" noises [19] (We use an approach in which the pump $E_{p}(t)$ is considered as a classical light field. Its quantum fluctuation is in fact hidden in the photo current shot noise.) 
A direct way of carrying out the "signal-noise" analysis would consist in calculation of output spectra densities and estimation of the SNR in some bandwidth after a matched filter. However, we will use in what follows the equivalent but more compact method in which all internal noises of the device fig. 1 are reduced to the input of the signal track fig.2. It is well known that an estimate of the input SNR corresponds to a SNR after the optimal filtering procedure.

We indicate with $N_{e}$ for the spectral density of the "read out noise" reduced to the fluctuation of DT coordinate $x_{2 n}$. Then the total input stochastic force can be written as

$$
F_{n}(t)=F_{T}(t)+K_{m}^{-1}(p) x_{2 n}(t) .
$$

where $F_{T}(t)$ is the equivalent stochastic force responsible for thermal noises. In a more general approach its spectrum density can be found through the fluctuation-dissipation theorem (FDT) [20]. According to this theorem $\left\langle\left|F_{T}(j \omega)\right|^{2}\right\rangle=2 k_{B} T \operatorname{Im}\left[K_{m}^{-1}(j \omega) / \omega\right]$, where $k_{B}$ is the Boltzmann's constant.. So the total input spectral density is described by the formula

$$
<\left|F_{n}(j \omega)\right|^{2}>=2 k_{B} T \operatorname{Im}\left[K_{m}^{-1}(j \omega) / \omega\right]+\left|K_{m}(j \omega)\right|^{-2} N_{e} .
$$

A "signal influence" is given by the well known equivalent "GW-force" perturbing the bar detector in the weak field approximation

$$
F_{g w}(t)=(1 / 2) m_{e q} L_{e q} p^{2} h(t) \sim\left(1 / \pi^{2}\right) M L \omega_{g}^{2} h_{0}
$$

Here $m_{e q}=M / 2$ and $L_{e q}=4 L / \pi^{2}$; the signal metric perturbation is supposed in the form of quasi resonance short pulse: $h(t)=h_{0} \sin \omega_{g} t, 0<t<\tau_{s}$ so that $\omega_{g} \sim \omega_{0}$ and $\omega_{g} \tau_{s} \sim 2 \pi$, i.e. a GW-pulse containing only a few periods of carrier, with a duration much shorter than the relaxation time of the bar.

In order to start with SNR analysis we need to define $N_{e}$.

\section{Equivalent optical read out noise}

Let us consider the physics of a FP sensor more in detail in order to clarify the read-out spectral density $N_{e}$. The three principal parameters of the cavity are the amplitude coefficients for mirror reflection $r$, transmission $\tau$ and absorption $a$ which satisfy the following relations

$$
r^{2}+|\tau|^{2}+a^{2}=1, \tau=j|\tau|
$$

The two FP cavity mirrors are supposed to be equal.

Let the complex amplitude of the laser pump be $\tilde{E}_{P}$. Then in a one mode approximation the amplitude of transmitted light $E_{\tau}$ is derived by the equation

$$
\begin{gathered}
\left(p^{2}+2 \gamma p+\omega_{n}^{2}\right) E_{\tau}=2 \gamma_{\tau} p E \\
E=2(-1)^{n} E_{P}, \omega_{n}=\pi\left(c / L_{0}\right) n, \gamma=\left(\gamma_{\tau}+\gamma_{a}\right), L=L_{0}+x_{2}
\end{gathered}
$$


Here $\omega_{n}$ is the resonance frequency of $n-t h$ optical mode of the cavity; the relaxation index $\gamma$ (half width of the resonance) has two contributions: one $\gamma_{\tau}=(c / 4 L)|\tau|^{2}$ due to light leaking from cavity; second $\gamma_{a}=(c / 4 L) a^{2}$ due to light absorption in the mirrors.

In a narrow region near the optical resonance $\omega_{P} \sim \omega_{n}$ the complex amplitude of the transmitted light $E_{\tau}$ can be approximated as

$$
\begin{gathered}
\tilde{E}_{\tau} \sim \frac{j \gamma_{\tau}}{(\Omega+\Delta-j \gamma)} \tilde{E}(j \omega), \\
\Omega=\omega-\omega_{n} ; \Delta=\omega_{P}-\omega_{n} \sim \omega_{n}\left(x_{2} / L\right) .
\end{gathered}
$$

The tuning at resonance $\Delta=0$, with the initial phase choice $\tilde{E}_{P}=E_{0}$, provides the signal amplitude of the transmitted light

$$
\tilde{E}_{\tau} \approx(-1)^{(n+1)} \frac{2 \gamma_{\tau}}{\gamma} E_{0} \exp \left\{-j \frac{\omega_{P}}{\gamma} \frac{x_{2}}{L}\right\}
$$

Thus the DT coordinate variation $x_{2}(t)$ produces a phase modulation of the transmitted light. The corresponding optical perturbation can be detected for example through the heterodyne photodetection with a reference field $E_{g}$ at the resonance frequency.

The only natural source of fluctuation in the approach of classical pump is a photo current shot noise $i_{n}(t)$

$$
\begin{gathered}
I(t)=I_{0}+i_{n}(t), I_{0}=\eta\left(e P_{g} / \hbar \omega_{n}\right) \\
<i_{n}(t) i_{n}(t+\tau)>=e I_{0} \delta(\tau)
\end{gathered}
$$

here we assume that the average current $I_{0}$ is produced mainly by the heterodyne power $P_{g}$; the symbols $e, \eta$ are used for the electron charge and the photo detector quantum efficiency.

Expanding the exponent (10) for small variation $x_{2}(t)$ one can get the equivalent current variation

$$
i(t) \approx I_{0} \frac{2 \gamma_{\tau}}{\gamma} \sqrt{\frac{P_{0}}{P_{g}}} \frac{\omega_{n} x_{2}(t)}{\gamma L}
$$

where $P_{0}$ is the transmitted light power. This formula allows us to consider the fluctuation $x_{2 n}(t)$ as an equivalent "read out noise" putting $i_{t}=i_{n}(t)$. The correlation function of this noise is

$$
\left\langle x_{2 n}(t) x_{2 n}(t+\tau)\right\rangle=\left(\frac{\gamma}{2 \gamma_{\tau}}\right)^{2} \frac{\hbar \omega_{n}}{\eta P_{0}}\left(\frac{\gamma L}{\omega_{n}}\right)^{2} \delta(\tau) .
$$

After substitution for the parameters $\left(\gamma, \gamma_{\tau}\right)$ one comes to the spectral density of the equivalent read out noise

$$
\left\langle\left|x_{2 n}(\omega)\right|^{2}\right\rangle=\left(\frac{\lambda}{4 \pi}\right)^{2}\left(|\tau|+\frac{a^{2}}{|\tau|}\right)^{4} \frac{\hbar \omega_{n}}{\eta P_{0}} .
$$

A minimum of this noise is achieved under the well known condition from the theory of FP sensors: the optimal tuning corresponds to equality of transmitted and absorbed 
parts of the light $|\tau|=a$. Finally the spectral density of "read out noise" looks like

$$
N_{e}=\left\langle\left|x_{2 n}(\omega)\right|^{2}\right\rangle=\left(\frac{\lambda}{2 \pi}\right)^{2}|\tau|^{4} \frac{\hbar \omega_{n}}{\eta P_{0}} .
$$

Just this expression has to be used in the formula (5).

\section{SNR analysis}

A knowledge of the signal properties (6) and noise spectrum (5) in term of equivalent input forces perturbing the opto-acoustical GW detector allows us to perform a signalnoise analysis in different conceivable cases.

\subsection{Potential sensitivity}

We shall call "potential sensitivity" a combination of amplitudes $F_{\min }, h_{\min }$ which can be registered in the "signal bandwidth" $\delta \omega=2 \pi / \tau_{s}$ by the bar detector with ideal (noiseless) read out sensor, i.e. only limited by the bar thermal noise in the entire signal bandwidth. The corresponding detection condition is

$$
\mathrm{SNR}=\rho_{0}=\frac{1}{4 \pi} \int_{-\infty}^{\infty} \frac{\left|F_{s}(j \omega)\right|^{2}}{2 k_{B} T H_{1}} d \omega=1
$$

For short signal pulses one can use the approximation $F_{s}(j \omega) \approx F_{0} \tau_{s}$. The substitution in the integrand (15) leads to the well known formulas

$$
F_{\min }=\sqrt{\frac{2 k_{B} T H_{1}}{\tau_{s}}}, \quad h_{\min }=\frac{2}{L}\left(\frac{k_{B} T}{m_{e q} \omega_{0}^{2}} \frac{1}{Q \omega_{0} \tau_{s}}\right)^{1 / 2}
$$

\subsection{Sensitivity of the opto-acoustical bar without DT}

We now consider, as an intermediate step toward the full calculation, the set-up in which the displacement transformer is absent and the mirror of the FP-cavity is attached directly to the bar, i.e. to the mass $\mathrm{M}$. The corresponding equations of motion can be reduced from (1) putting $m=0, H_{2}=0, F_{f l 2}=0$.

The input noise spectral density will look as

$$
<\left|F_{n}(j \Omega)\right|>=2 k_{B} T H_{1}\left[1+\left(\Omega / \Omega_{r}\right)^{2}\right]
$$

where

$$
\Omega_{r}^{2}=\frac{2 k_{B} T H_{1}}{4 \pi M^{2} \omega_{0}^{2} N_{e}}=\frac{k_{B} T}{M \omega_{0}^{2}} \frac{\omega_{0}}{2 \pi Q N_{e}} ; \Omega=\omega_{0}-\omega,|\Omega| \ll \omega_{0} .
$$

(to derive this formula, a usual approximation, typical for the near resonance zone, was used : $\left.\left[\left(\omega^{2}-\omega_{0}^{2}\right)^{2}+4 \delta^{2} \omega^{2}\right] \approx 4 \omega_{0}^{2}\left(\Omega^{2}+\delta^{2}\right), \delta \ll|\Omega| \ll \omega_{0}\right)$.

Looking at the noise spectrum (17) one can conclude that the potential sensitivity (16) will be kept around resonance frequency $\omega_{0}$ in the bandwidth $\pm \Omega_{r}$ defined by the ratio of intensities of the thermal and optical (read out) noises. 


\subsection{Real sensitivity of the total scheme}

In practice, a DT is used to increase an amplitude of the detected displacement and therefore, beside the read out noise, also the thermal noise of DT will limit the sensitivity. To estimate it one can use the general formula for input noise spectrum (5). The special interest of our calculation as in above consists in estimation of the frequency band inside of which the sensitivity would be kept on its potential level (16).

Using formulas (14), (5) and (2) one can get the following result for the input spectral noise density

$$
\begin{gathered}
\left\langle\left|F_{n}(j \Omega)\right|^{2}\right\rangle=2 k_{B} T H_{1} \Gamma(\Omega) \\
\Gamma(\Omega) \approx 1+\frac{\left(\Omega^{2}-\Omega_{B}^{2}\right)^{2}}{\omega_{r}^{4}}+4 \varepsilon^{-1} \frac{Q_{1}}{Q_{2}}\left(\frac{\Omega+\Omega_{0}}{\omega_{0}}\right)^{2} .
\end{gathered}
$$

This expression for the noise factor $\Gamma(\Omega)$ is approximately valid in a region near the resonant frequency $|\Omega| \ll \omega_{0}$. A specific parameter $\omega_{r}^{2}=\omega_{0} \Omega_{r} / 2=\omega_{0}^{2}\left(\Omega_{r} / 2 \omega_{0}\right)$ presents a normalized value of the bandwidth $\Omega_{r}$. Other symbols in (19) are: $\Omega_{0}=$ $\omega_{0}-\omega_{1},\left|\Omega_{0}\right| \ll \omega_{0}$ is the initial detuning and $\mu^{-1}\left(Q_{1} / Q_{2}\right) \approx H_{2} / H_{1}$ is the ratio of quality factors of the bar $Q_{1}$ and DT $Q_{2}$ associated with the corresponding losses.

In analogy with the points $\mathrm{A}, \mathrm{B}$ above, one can write down the expression for SNR using the noise spectrum (19). The potential sensitivity will be kept only in the frequency zone where the two last terms in eq.(19) do not exceed unity. Without loss of generality, it is convenient to consider a very practical case of equal partial frequencies $\omega_{1}=\omega_{2}$. Then the initial detuning is absent: $\Omega_{0}=0$. Thus, requiring that the excess noise factor $\Gamma^{+}(\Omega)=\Gamma(\Omega)-1$ be small leads to the inequality

$$
\Gamma^{+}(\Omega)=\frac{\left(\Omega^{2}-\Omega_{B}^{2}\right)^{2}}{\omega_{r}^{4}}+4 \mu^{-1} \frac{Q_{1}}{Q_{2}}\left(\frac{\Omega}{\omega_{0}}\right)^{2} \leq 1
$$

It is interesting to note that (20) can be presented in an oscillatory form:

$$
\Gamma^{+}(\Omega)=\omega_{r}^{-4}\left|K_{e}(j \Omega)\right|^{-2}, K_{e}(j \Omega)=\left(\Omega_{B}^{2}-\Omega^{2}+2 j \gamma_{e} \Omega\right)^{-1},
$$

where $K_{e}(j \Omega)$ is the transfer function of a low-frequency equivalent oscillator having the resonance at the beating frequency $\Omega_{B}$ with the quality factor

$$
Q_{e}=\frac{\Omega_{B}}{2 \gamma_{e}}=\left(\frac{\Omega_{B}}{\omega_{r}}\right)^{2} \sqrt{\frac{Q_{2}}{Q_{1}}} .
$$

Such new description of the excess noise factor $\Gamma^{+}(\Omega)$ allows to perform analytically a choice of key parameters of the problem adjusting the best "sensitivity- bandwidth" relation.

There are two different types of behavior of the system: the so called "oscillation regime" for $Q_{e}>1 / 2$ and the "relaxation regime": $Q_{e}<1 / 2$. The difference in these two regimes is reflected on the character of "zones of effective sensitivity". 
A solution of eq.(20) lets us define the frequency range of such zones. It is convenient to use the following dimensionless variables:

$$
x=\left(\frac{\Omega}{\Omega_{B}}\right)^{2}, \xi=\left(\frac{\omega_{r}}{\Omega_{B}}\right)^{4}, 2 \nu=\left(\frac{Q_{1}}{Q_{2}}\right) .
$$

then the inequality (20) is read as

$$
(x-1)^{2}+2 \xi \nu x-\xi \leq 0 .
$$

with roots

$$
x_{1,2}=(1-\xi \nu) \pm \sqrt{D}, \quad D=(1-\xi \nu)^{2}+\xi-1, \quad\left(\xi, x_{1,2}>0\right),
$$

and the evident constraint on values of free parameters $\xi, \nu$

$$
D \geq 0: \xi \geq(2 \nu-1) / \nu^{2} .
$$

As one can see from (23) the determinant $D$ is sensitive to variations $\xi$ around the threshold $\xi=1$ separating two different type of solutions.

a). The "relaxation regime" $\xi>1$.

There are two real roots of the equation (22) but only the positive one is acceptable. Then the inequality (22) is fulfilled in the one central zone of "potential sensitivity"

$$
0 \leq x \leq(1-\xi \nu)+\sqrt{D} .
$$

or coming back to frequencies (21) one estimates the width of this zone as

$$
\Delta \Omega \leq 2 \Omega_{B} \sqrt{(1-\xi \nu)+\sqrt{D}},
$$

b). The "oscillation regime" $\xi<1$.

Positive roots of the equation (22) exist only if $\xi<1 / \nu$ (23) (we remark that eq. (24) is contradicted if $\nu>1$, so such regime is possible only for $\nu<1$ ). In this case a solution of the inequality (22) results in

$$
(1-\xi \nu)-\sqrt{D} \leq x \leq(1-\xi \nu)+\sqrt{D} .
$$

It means there are two permitted frequency zones symmetrical with respect to the central frequency $\omega_{0}$ with a total width given by

$$
\Delta \Omega \leq \Omega_{B} \frac{4 \sqrt{D}}{\sqrt{(1-\xi \nu)+\sqrt{D}}+\sqrt{(1-\xi \nu)-\sqrt{D}}} .
$$

In the limiting case $\xi \rightarrow 1$ two back side zones are transformed into the single central one (25). 


\section{Numerical estimates}

Now we can return to the main target of our analysis - an estimation of the resonant antenna bandwidth in which the sensitivity might be kept at the potential level, limited only by the bar thermal noise.

Our interest to get such estimate for two variants of bar antennas: first, the room temperature bar with DT and FP-optical read out (Russian project OGRAN); second, a supercryogenic bar with DT using FP-optical read out instead of the SQUID sensor.

Preliminarily, we have to numerically define the level of potential sensitivity in term of spectral noise density for both cases of interest according to eq. (16). Typical parameters of bars are the following: the effective length and mass $-L \approx 2 m, M \approx$ $10^{3} \mathrm{~kg}$, the resonance frequencies $-\omega_{0}=8 \cdot 10^{3} \mathrm{~s}^{-1}(\mathrm{OGRAN})$ and $\omega_{0}=5.8 \cdot 10^{3} \mathrm{~s}^{-1}$ (Nautilus), the temperature $T=300 \mathrm{~K}$ (OGRAN) and $T=10^{-1} \mathrm{~K}$ for Nautilus (although recent results on the Minigrail detector [21] show that $T=10^{-2} \mathrm{~K}$ is attainable), the quality factors $-Q=1.6 \cdot 10^{5}$ (OGRAN) and $Q=6 \cdot 10^{6}$ (Nautilus).

Substitution of these values into eq. (16) leads to estimates of the "potential sensitivity"

$\alpha)$ for room temperature detector (OGRAN):

$$
|h(f)|_{n} \approx 1.5 \cdot 10^{-20} \mathrm{~Hz}^{-1 / 2}, h_{\min } \approx 4.5 \cdot 10^{-19} .
$$

$\beta$ ) for super cryogenic detector (Nautilus):

$$
|h(f)|_{n} \approx 10^{-23} \mathrm{~Hz}^{-1 / 2}, h_{\min } \approx 3 \cdot 10^{-22} .
$$

In both cases $h_{\min }$ was estimated in the the signal band: $\Delta f=\tau_{s}^{-1}=10^{3} \mathrm{~Hz}$, as we are dealing with "potential sensitivity".

We then introduce the effect of readout. Assuming for the optical readout system the following parameters: external infrared laser power $P=1 \mathrm{~W}(\lambda=1 \mu \mathrm{m})$ and finesse of FP resonator $\mathcal{F} \approx \pi /(1-R)=3000$, one can calculate the admitted frequency bandwidth of corresponding gravitational antenna.

i) Room temperature opto-acoustical bar with DT (OGRAN).

At room temperature it is technically difficult to get equal quality factors $Q_{1} \approx Q_{2}$. In practice quality factor of the DT is usually much less the bar one $Q_{2} \ll Q_{1}$ [15] and so $\nu=\left(Q_{1} / Q_{2}\right) \gg 1$. It means a such detector can operate only in the "relaxation regime" $\xi>1$. Then, taking a limit of $(25)$ under the condition $\xi \nu \gg 1$ we come to the estimate of effective detection bandwidth

$$
\Delta \Omega \leq 2 \Omega_{B} \sqrt{1 / 2 \nu}=2 \Omega_{B} \sqrt{Q_{2} / Q_{1}}=\omega_{0} \sqrt{\mu Q_{2} / Q_{1}}
$$

Thus the effective bandwidth $\Delta \Omega$ is defined by the parameter of losses $\nu=\left(Q_{1} / 2 Q_{2}\right) \gg$ 1 and the beating frequency $\Omega_{B}$, i.e. the main limiting role belongs to the thermal noise of DT. However this regime can be realized only with a good optical sensor with 
sufficiently small read out noise. Indeed, the beating frequency $\Omega_{B}$ here is limited by the condition $\xi>1$ or in the equivalent form $\Omega_{B}<\omega_{r}=\sqrt{\omega_{0} \Omega_{r} / 2}$, where (see (18), (14))

$$
\Omega_{r}^{2}=\left(\frac{\pi \mathcal{F}}{\lambda}\right)^{2} \frac{k_{B} T}{M \omega_{0}^{2}} \frac{\omega_{0}}{2 \pi Q_{1}} \frac{\eta P_{0}}{\hbar \omega_{n}} .
$$

In this formula the finesse of FP-sensor was taken as $\mathcal{F}=2 \tau^{-2}$. By substituting the values $\mu \simeq 10^{-2}$ and $Q_{1} / Q_{2} \simeq 30$ we come to the estimate: $\Delta \Omega \simeq 0,02 \omega_{0}$.

ii) Cryogenic detector with optical read out.

At low temperature the DT quality factor can be large enough, $Q_{2} \sim Q_{1}, \nu=1 / 2$. Therefore, the estimates of effective bandwidth for two cases of interest take the form:

$$
\begin{gathered}
\xi>1: \Delta \Omega \leq 2 \Omega_{B} ; \\
\xi<1: \Delta \Omega \leq 2 \Omega_{B} \frac{\xi}{1+\sqrt{1-\xi}}
\end{gathered}
$$

Analysis of the formulae (28), (30a),(30b) shows that:

i). A maximum value of the detection bandwidth $\Delta \Omega$ takes place in the "relaxation regime" and it is defined by the beating frequency $\Omega_{B}$. Optimal choice of parameters corresponds to the condition

$$
\Omega_{B}=0.5 ; \quad \sqrt{\mu} \omega_{0} \approx \omega_{r} ; \quad \xi \sim 1, \quad \Omega_{r}=0.5 \mu \omega_{0} .
$$

These relations define the requirements for the parameters of the optical sensor $\left(P_{0}, \mathcal{F}\right)$ for a given transformer factor $\mu=m / M$.

ii).In the "oscillation regime" $\left(\Omega_{B}>\omega_{r}\right)$ the effective detection bandwidth is decreased in the factor $(1+\sqrt{1-\xi}) / \xi$ but it is not too important for the practical value of $\xi \sim 1$.

An estimate of the effective detection bandwidth for a cryogenic bar with the optimal optical read out yields: $\Delta \omega=\sqrt{\mu} \omega_{0} \simeq 0.1 \omega_{0}$ i.e. the potential sensitivity of a bar with resonance frequency close to $1 \mathrm{kHz}$ can be realized in the frequency region on the order of hundred Hz. Typical frequency bandwidth variations versus $\xi, \nu$ parameters are illustrated by fig.3; the left column corresponds to the room temperature setups, the right column to the cryogenic ones.

\section{Discussion and conclusions}

Our analysis has shown that a room temperature bar detector equipped with a DT with lower quality factor can actually achieve the limit sensitivity (limited only by the thermal noise of the bar) despite the presence of dominant DT thermal noise. The

physical reason of this is the effect of dynamical damping of DT noises in a narrow region near the partial bar frequency $\omega_{0}$. The depth and width of the damping is proportional 
to the bar quality factor and to the coupling parameter $\mu$. However, the width of such damping or the "width of potential sensitivity zone" is invariably smaller than the width that the same zone would have if no DT were used (see fig.3). A correspondent condition can be written as

$$
\frac{Q_{2}}{Q_{1}} \leq\left(\frac{\Omega_{r}}{\Omega_{B}}\right)^{2}
$$

If the inequality (31) is fulfilled there is no advantage to use DT.

If we use the parameters of the OGRAN project: $P=100 \mathrm{~mW}, T=300 \mathrm{~K}$, $\mathcal{F}=3 \cdot 10^{3}, Q_{1}=1.64 \cdot 10^{5}, Q_{1} / Q_{2}=30, \mu=10^{-2}, \eta=0.5$, the bandwidth defined by the optical FP sensor is $2 \Omega_{r} \sim 2.8 \cdot 10^{2} \mathrm{rad} / \mathrm{s}$. Application of the corresponding DT reduces the bandwidth to $\Omega_{r} \sim 1,5 \cdot 10^{2} \mathrm{rad} / \mathrm{s}$, i.e. results in almost halving it.

As an example, consider the case of the test experiment with the optical bar prototype of the AURIGA group [15]: $P=1.5 \mathrm{~mW}, \mathcal{F}=2.8 \cdot 10^{4}, Q_{1}=1.8 \cdot 10^{5}$, $Q_{1} / Q_{2}=27, \omega_{0}=5.45 \cdot 10^{3} \mathrm{rad} / \mathrm{s}, \mu=1.7 \cdot 10^{-3}$; the reduction would consist of almost one order of magnitude. The bandwidth of potential sensitivity achieved in the experiment [15] $\sim 43 \mathrm{rad} / \mathrm{s}$ with DT and a short FP cavity $(\sim 2 \mathrm{~cm})$ could be expanded up to $400 \mathrm{rad} / \mathrm{s}$ without DT. But in this case the optical read out should be realized through a long FP cavity with mirrors attached to the bar ends. Just this technical ability,- a central tunnel along the bar axis- is foreseen in the OGRAN project.

The realistic value of the bandwidth of potential sensitivity planned for the OGRAN project [3] consists of a few dozens Hertz.

For a supercryogenic bar with the physical temperature $T=10 \mathrm{mK}$ and a high quality DT $Q \approx 10^{7}$ the potential spectral noise density $\approx 10^{-23} \mathrm{~Hz}^{-1 / 2}$ can be achieved in the bandwidth a hundred $\mathrm{Hz}$ with optical sensor of moderate parameters $P_{0} \simeq(0.1-1.0) W, \mathcal{F} \sim\left(10^{4}-3 \cdot 10^{3}\right)$.

A more conservative estimate of sensitivity $\sim 310^{-21} \mathrm{~Hz}^{-1 / 2}$ and bandwidth $50 \mathrm{~Hz}$ for cryogenic bar with a small gap optical sensor operated as an accelerometer can be found in ref.[22]. However it was limited by technical noises described in that paper. In particular the additive technical laser power noise was included and it was taken a factor 200 larger than DT thermal noise. The temperature $T=0.1 \mathrm{~K}$ was 10 times larger than our estimation above. Finally, the product of important parameters, crucial for increasing sensitivity $P L_{t}^{2} \mathcal{F}^{2}$ (laser power, DT length, DT cavity finesse) was a factor 40 less. So the total noise power gain turns out to be of the order of $10^{4}$ in favor of our scheme. It results in a factor 300 for the sensitivity estimate in term of metric perturbation. The frequency spectral density noise estimation has the same order of value in both cases. Other technical noises of a PZ-actuator and its driver used in the paper [22] did not exceed the frequency noise (with the parameters of the AURIGA setup).

It worth to remark here the advantage of the FP sensor with a small cavity gap is known as a method of suppressing laser technical frequency fluctuation. Regarding laser power fluctuation, the situation is opposite: a corresponding frequency noise (induced by power variations) is inversely proportional to the cavity length. In that respect, an 
OGRAN cavity as long as the gravitational bar scale looks preferable.

In this paper we only focused on fundamental natural fluctuation: thermal mechanical noises and optical short noise, neglecting technical noises. However, the Virgo and Ligo experiments have proven that technical noises of the laser pump, potodetectors, mirror drivers etc. can be sufficiently reduced, at least in the kilohertz frequency region. so that the light power fluctuation foreseen in the Virgo interferometer does not exceed the corresponding shot noise level $\left|\left\langle\Delta P_{f}^{2}\right\rangle\right|^{1 / 2} \sim 10^{-10} \mathrm{WHz}^{-1 / 2}$ We believe that further increases of laser power and mirror finesse will be available in the near future. This would allow to further expand the detection bandwidth up to $10^{3}$ $\mathrm{Hz}(27 \mathrm{~b})$ (in our analysis we could not calculate it due to our restriction of "close to resonance approximation" (17),(19)).

One serious problem we encounter on this way is matching the optical cavity with power pump when dealing with mirrors cooled to very low temperatures. In fact, at present this problem has not yet a definite experimental solution. Investigation of cooled mirrors with incident and reflected optical power on the order of $1 \mathrm{~W}$ (and more) is carried out now by interferometer groups as advanced setups working at cryogenic temperature are planned. As an example, the well known Japanese LCGT-project [23] is designed for operation with mirrors cooled at $20 K$ and $100 \mathrm{~W}$ light power. Preliminary experiments have shown that mirrors with absorption $1 \mathrm{ppm}$ and power $0.1 \mathrm{~W}$ cooled to $4 \mathrm{~K}$ do not increase a mechanical and optical noise level typical for present generation of interferometers. There are indeed no experiments with mirrors at supercryogenic temperature. However we believe that the thermal contact of the mirrors with cryostat in the case of the "bar-interferometer" will be much better than for suspended mirrors (free mass interferometer). Recent advances in dilution refrigeration technology have made commercially available [24] devices with large cooling power at very low temperatures (up to $50 \mu W$ at $30 \mathrm{mK}$ ). So, the problem of mantaining the low temperature of mirrors illuminated by the light beam will probably reduce to proper design of the cryostat.

Our calculation above was performed also under the simplification of "zero detuning" $\omega_{0}=\omega_{1}$. Non perfect tuning produces a mismatch and in principle destroys the balance of noises. But if such detuning does not exceed the beating frequency all results formulated above remain valid.

It worth to remark that the reception band of GW bars might be expanded through a parametrical regeneration of the DT oscillator. Theoretically this possibility was considered in the paper [25]. The electrical rf-resonance circuit $\left(\omega_{0}\right)$ coupled with the DT having a DC bias can be regenerated by applying also AC pump at the double frequency $\left(2 \omega_{0}\right)$. The regeneration energy is regulated by alternative amplitude of the pump at some so called "frequency of superization" $\Omega_{s p} \ll \omega_{0}$ (see details in [25]). Finally, it leads to an equivalent increase the reception bandwidth and transfer function. However the application of this method to the DT with optical FP cavity has to be specifically addressed.

As a further development, we are considering with interest a set-up with each mirror of the FP mounted on a separate DT, thus fully exploiting the long baseline feature of 
this readout. In this case, the so called Janus scheme [26], we deal with a 3 mode mechanical system (bar + two DTs on opposite end faces) that has also very interesting symmetry properties with respect to a real g.w. signal : indeed, of the three resulting normal modes, the central one has no quadruple moment, and can then be used as an effective internal veto. Further analysis, needed to characterize this setup, is in progress.

\section{Acknowlegments}

The authors would like gratitude their colleagues professors G.Pizzella , G.Pallottino, F.Ricci (Universities of Roma), E.Majorana (INFN) and also S.Vyatchanin, V.Mitrofanov (MSU) for useful discussions while preparing this paper.

\section{References}

[1] P.Astone et al// Phys. Rev. D, v.68, p. 022001 (2003); arXiv: gr-qc/0705.0688v1. 4 May 2007.

[2] http: www.ligo.org; http: www.virgo.infn.it.

[3] L.Bezrukov, S.Popov, V.Rudenko et.al."Gravitational Wave Experiments and Baksan project OGRAN", pp. 125 in the book: Astrophysics and Cosmology after Gamov. Cambridge Sci. Publ.,2007

[4] P.Astone, M.Bassan, P.Bonifazi et.al. Phys.Rev.D v.47,N2,p.362-375, 1993.

[5] P.Astone, M.Bassan, D.Babusci et.al. Astroparticle Physics,v.7,pp.231-240, 1997.

[6] J.P.Zendri et.al., Class.Quant.Grav. v.19,p.1925, 2002.

[7] H.J.Paik, J.Appl.Phys. v.47, pp.1168-1178, 1976.

[8] P.Michelson, R.C.Taber, Phys.Rev.D v.29,(N10),pp.2149-2152,1984

[9] V.V.Kulagin, A.G.Polnarev, V.N.Rudenko. Sov.Phys.JETP v.64, iss.5,pp.9115-9121, 1986.

[10] J.P.Richard. J.Appl.Phys.,v.64, pp.2202-2205, 1988.

[11] J.P.Richard. Phys.Rev.D,v.46,2309 1992.

[12] N.Mio, K.Tsubono. Appl.Optics, v.34, N1, pp. 186-189, 1995.

[13] Yi Pang and J.P.Richard. Appl.Opt.,v.34,4982, 1995.

[14] A.V.Gusev, V.V.Kulagin, V.N.Rudenko. Gravitation \& Cosmology, v.2, N1(5), pp.68-70, 1996 (published by Russ.Grav.Society, ISSN 0202-2893)

[15] M.De Rosa, L.Baggio, M.Cerdonio, L.Conti et.al. Class.Quantum Grav.,v.19,pp.1919-1924, 2002 ; also L.Conti, M.De Rosa, F.Marin, T.Taffarello et.al. J.Appl. Phys.,v.93,p.3589, 2003.

[16] V.V.Kulagin, V.N.Rudenko. Phys.Lett.A 214, pp.123-126,1996.

[17] A.Di Virgilio, L.Barsotti, S.Braccini et.al.., Phys.Rev.A. v.74, p.013813,2006.

[18] L.D.Landau, E.Lifshits. Theory of elasticity, (Theoretical Physics VII), Butterworth-Heinemann 1986 (3rd edition)

[19] R.M.Gagliardi, S.Karp. Optical communications. A Wiley-Interscince Publication. John Wiley \& Sons, 1976. New York, London, Sydney,Toronto.

[20] L.D.Landau, E.M.Lifshits. Statistical Physics (Theoretical Physics V), Butterworth-Heinemann 1984 (3rd edition)

[21] A de Waard, L.Gottardi, G.Frossati. Class.Quant.Grav. v.19, p.1935, 2002.

[22] L.Conti, M.Cerdonio, L.Traffarello, J.P.Zendri et.al. Rev.Sci.Instrum. V.69,n2,p.554-558,1998.

[23] K.Kuroda,M.Ohashi,M.Miyoki et.al. Int.J.Mod.Phys.D v.8,n5,p.557,1999.

[24] http://www.leidencryogenics.com

[25] A.V.Gusev, V.N.Rudenko. Phys. Letters A 175, pp. 382 - 386, 1993

[26] M.Canzoniere, E.Majorana, Y.Ogawa et.al. Phys.Rev.D47,N12,pp.5233-5237, 1993. 


\section{Appendix}

A critical magnitude of laser power is defined by an equality of the thermal noise of mirrors and its fluctuation under the light photon pressure. The pressure force is described as stochastic series of photon shots

$$
f(t)=(1+R) \frac{\hbar \omega_{n}}{c} \sum_{\nu} \delta\left(t-t_{\nu}\right)
$$

which is considered as the Poissonian process with moments

$$
\langle f(t)\rangle=\frac{\hbar \omega_{n}}{c} N_{1},\langle\tilde{f}(t) \tilde{f}(t+\tau)\rangle=\left(\frac{\hbar \omega_{n}}{c}\right)^{2} N_{1} \delta(\tau) .
$$

Here $N_{1}=P / \hbar \omega_{n}$ is the average rate of photons inside the FP cavity; $P=P_{0} /(1-R)$, $P_{0}$ is the external laser power.

Thus the correlation function of the light pressure force read as

$$
\langle\tilde{f}(t) \tilde{f}(t+\tau)\rangle=(1+R)^{2}\left(\frac{\hbar \omega_{n}}{c}\right)^{2} N_{1} \delta(\tau) \approx 4 \frac{\hbar \omega_{n} P_{0}}{(1-R) c^{2}} \delta(\tau) .
$$

It has to be compared with the Nyquist force correlation function $\left\langle f_{T}(t) f_{T}(t+\tau)\right\rangle=$ $2 k_{B} T H \delta(\tau)$. Finally the critical laser power is given as

$$
\left[P_{0}\right]_{\mathrm{cr}}=4\left(\frac{k_{B} T}{\hbar \omega_{n}}\right) \frac{m \omega_{0}}{Q \mathcal{F}} c^{2} .
$$

Substitution typical parameters: $T=10 \mathrm{mK}, \lambda=1 \mu \mathrm{m}, m=10^{4} \mathrm{~g}, \omega_{0} \approx 6 \cdot 10^{3} \mathrm{rad} / \mathrm{s}$, $Q \approx 10^{7}, \mathcal{F} \approx 10^{4}$ results in the estimation $\left[P_{0}\right]_{\mathrm{cr}} \approx 160 \cdot 10^{3} \mathrm{~kW}$ i. e. too large a value for a practical laser pump. 


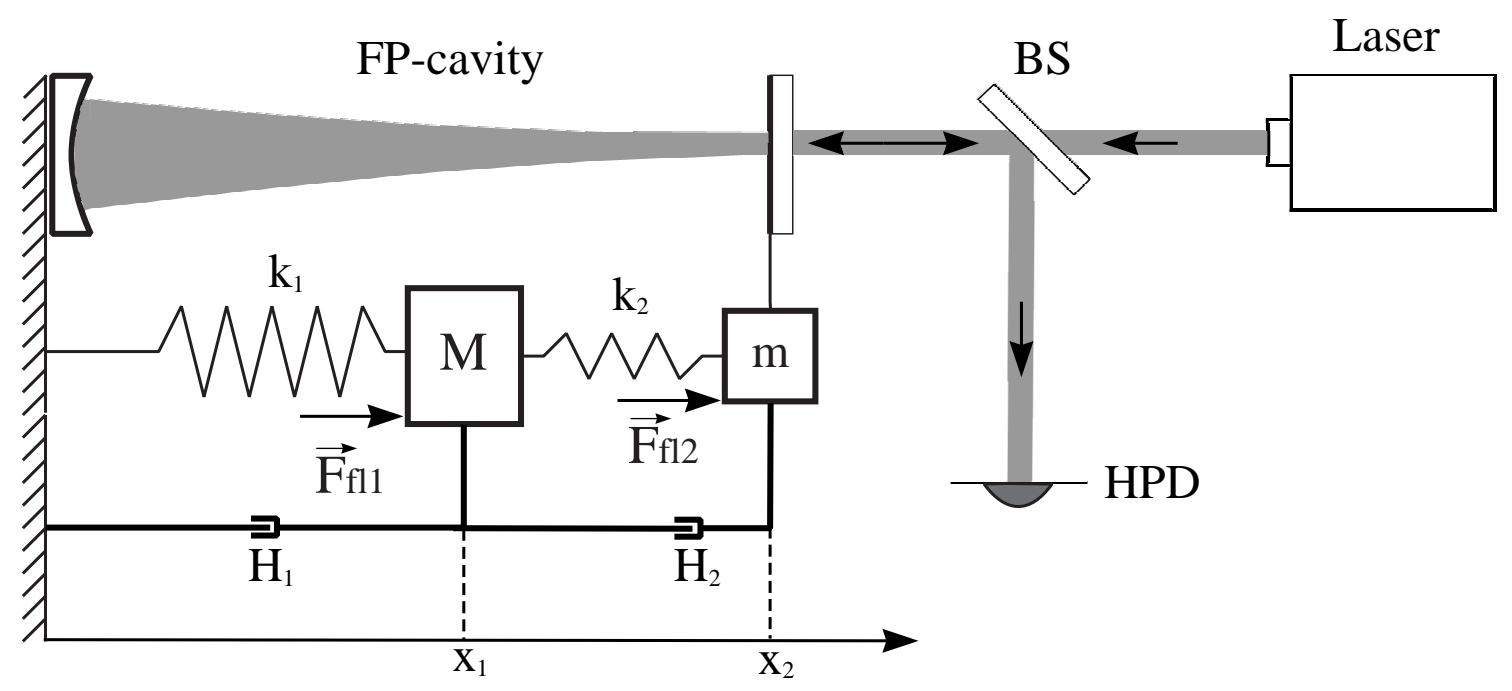

Figure 1. Equivalent scheme of the opto-acoustical detector. $M, k_{1}, H_{1}$ - mass, rigidity and friction coefficient of the bar. $m, k_{2}, H_{2}$ - correspondent transducer parameters.

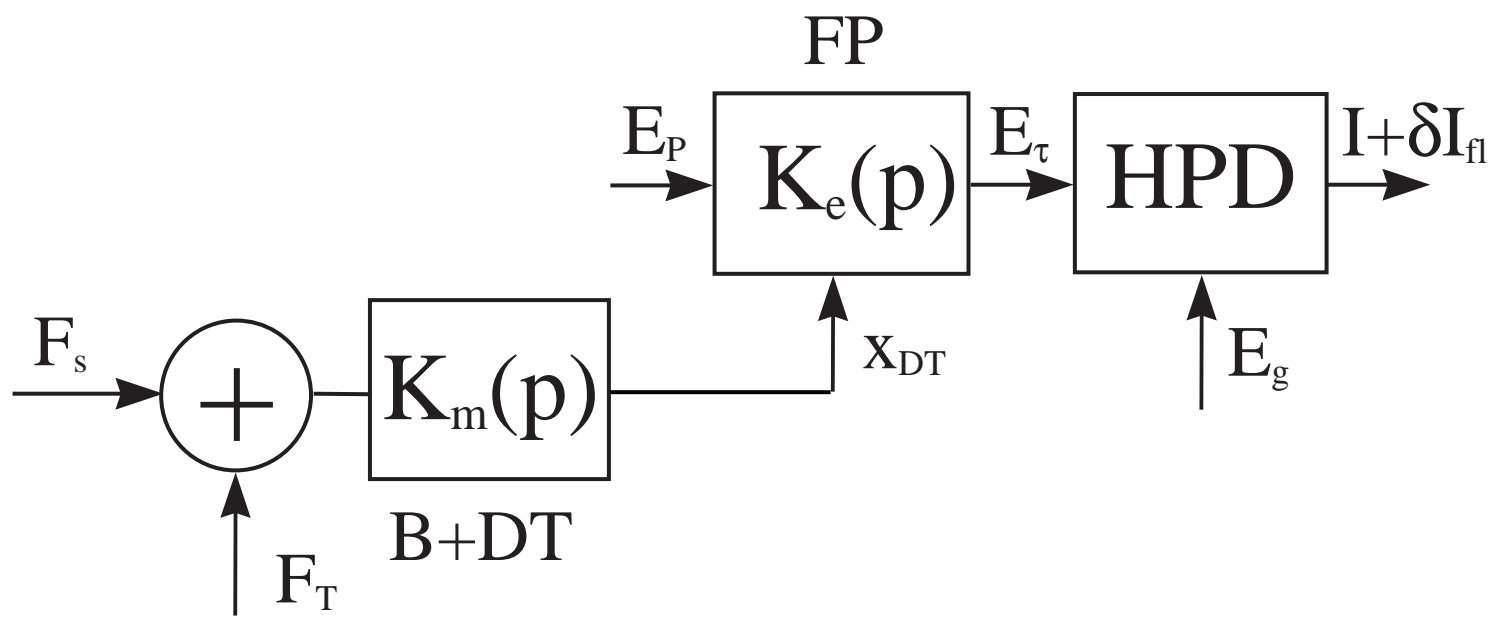

Figure 2. Block diagram of the signal/noise track. $K_{m}(p), K_{e}(p)-$ mechanical and optical transfer functions of the setup. HPD $=$ a heterodyne photo detector 

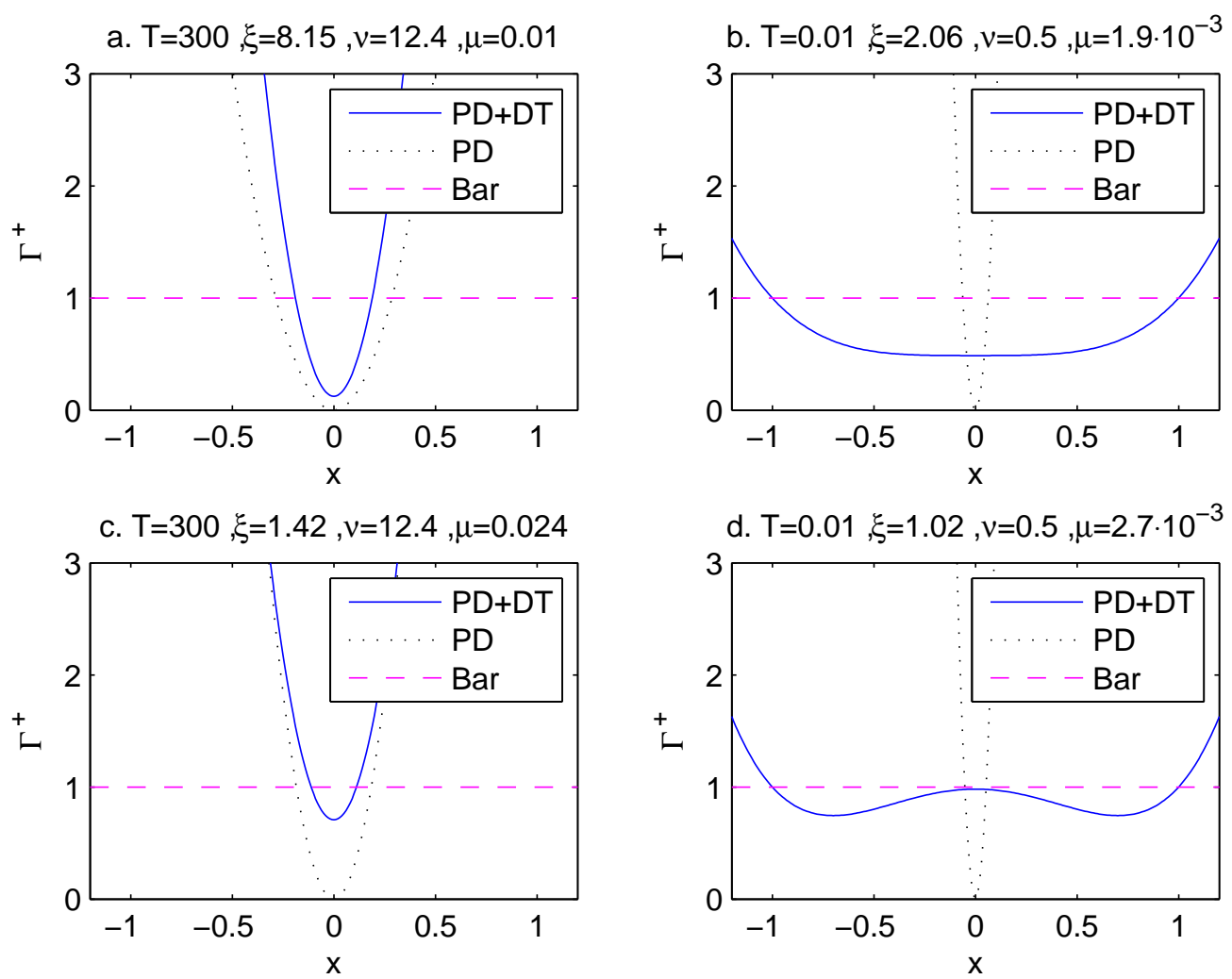

e. $\mathrm{T}=300, \xi=0.906, v=12.4, \mu=0.03$

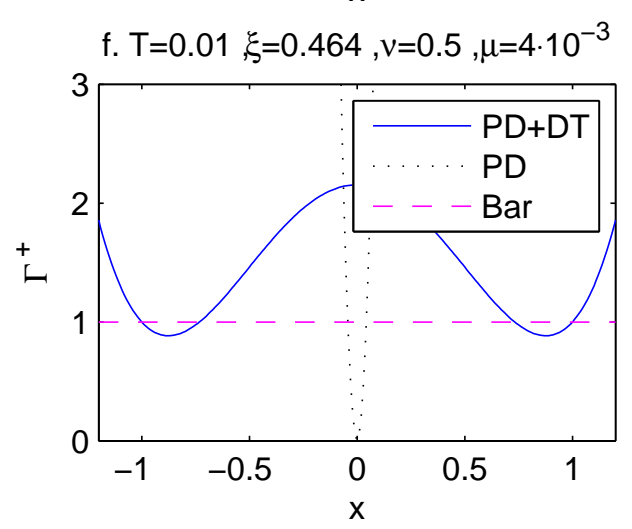

Figure 3. Budget of noises $\Gamma^{+}=<\left|F_{n}(j \omega)\right|>2 k_{B} T H_{1}$ versus parameters $\nu, \xi(\nu=$ $\left.Q_{1} / 2 Q_{2} ; \xi=\omega_{r} / \Omega_{B}\right), x=\Omega / \Omega_{B}$ PD-optical readout only, PD+DT - optical readout with transducer. Dashed line is bar noise 